\title{
Application des techniques d'antennerie à la reconstruction du déplacement d'une structure vibrante
}

\author{
P.-O. MATTEI et P.J.T. FILIPPI
}

Laboratoire de Mécanique et d'Acoustique, 31 Chemin Joseph-Aiguier, 13402 Marseille cedex 20, France

\begin{abstract}
Résumé : On s'interesse au problème de 1'identification du déplacement d'une plaque mince vibrante à partir de mesures de pression acoustique. On utilise une interpolation analytique entre les points de mesure, issue d'une décomposition du champ acoustique en série d'harmoniques sphériques, pour améliorer 7 'identification du déplacement de la plaque, modélisé par représentation de Green. Des résultats expérimentaux sont comparés à une solution analytique. La méthode est appliquée à la détection d'un défaut.

Abstract : One considers the problem of the identification of the displacement of a thin vibrating plate from measurement of the acoustic radiated pressure. The use of an analytical interpolation, obtained by a decomposition of the acoustic sound pressure into a series of spherical harmonics, gives a better identification of the displacement of the plate. modelized by a Green representation. Numerical results are compared with an analytical solution. The method is applied to the detection of a defect.
\end{abstract}

\section{INTRODUCTION}

On présente dans cette communication quelques aspects des techniques d'antennerie appliquées à la reconstruction du déplacement d'une structure mince qui vibre en ambiance anéchoïque. L'objectif vise, non seulement identifier la déformée de la structure en régime harmonique, mais encore à détecter la présence d'un défaut, ici une discontinuité de masse. L'idée qui est développée repose sur une double identification de la pression acoustique que rayonne la plaque. Dans un premier temps. on cherche à identifier la décomposition en série d'harmoniques sphériques [1]. Dans une deuxième phase, une représentation de Green de la pression rayonnée par la plaque est introduite sous 1 a forme d'un potentiel de double couche. De part la géométrie circulaire du problème, ce potentiel est représenté sous forme d'une série de Fourier angulaire dont les coefficients sont cherchés sous la forme de polynômes de Tchebycheff. L'intérêt de cette double identification tient au fait que les harmoniques sphériques sont des fonctions qui satisfont 1 'équation de propagation du son, ainsi, le champ acoustique est identifié avec une très bonne précision avec peu de mesures. Il est alors possible de construire une interpolation du champ de acoustique mesuré qui comporte un nombre bien plus important de "points de mesure". Comme, généralement, l'équation de propagation des ondes mécaniques n'est pas connue. il est nécessaire de disposer de beaucoup plus de mesures pour identifier correctement la série qui représente le déplacement de la structure. Cette méthode présente une grande souplesse d"utilisation puisque le nombre de "mesures" dont on dispose est adaptée, à très faible coût numérique, aux besoins de 1'identification du déplacement. 


\section{DESCRIPTION DU DISPOSITIF EXPERIMENTAL}

On considère une plaque en acier circulaire de $20 \mathrm{~cm}$ de rayon et de $1 \mathrm{~mm}$ d'épaisseur. Son module d'young est de $2010^{11} \mathrm{~Pa}$ et son coefficient de Poisson $v=0,3$. Cette plaque est excitée par des signaux harmoniques par un pot vibrant fixé en son centre par l'intermédaire d'une tige rigide. Un cadre metallique supporte les microphones de mesure et entoure la plaque. Sur cette structure, 32 microphones ont été disposés régulièrement sur une sphère qui entoure la plaque. Un dernier microphone, dit "microphone de référence" est fixé au cadre métaliqque. L'ensemble du montage est placé en salle anéchoique. Pour diminuer le coût du montage, nous nous sommes contentés de capteurs dont les performances sont modestes ma is stables au cours de la mesure. Tous ces capteurs sont préalablement étalonnés par rapport à un microphone de haute qualité. Ceci permet de disposer de données d'amplitude et de phase absolue. Ces étalonnages permettent de convertir les données brutes en données exactes. Après acquisition des signaux, l'ensemble des mesures est ramené à la valeur de la pression donnée par le microphone de référence.

\section{RAPPELS THEORIQUES}

\subsection{Modélisation d'un champ acoustique à l'aide d'une série d'harmoniques sphériques}

On considère une structure vibrante qui occupe un domaine $\Omega$, de frontière $a \Omega$. Cette source rayonne en espace indéfini une pression acoustique harmonique $\left(e^{-i \omega t}\right)$. On nomme $\Psi(M)$ ce champ. Soit 0,7 'origine d'un système de coordonnées sphériques $(0, \vec{r}, \vec{\theta}, \vec{\varphi})$. Soit $\Sigma_{0}$, la plus petite sphère, centrée sur 0 , qui contient $\Omega$. Il est tout à fait classique qu'à l'extérieur de $\Sigma_{0}, \psi(M)$ peut être développé sous la forme suivante :

$$
\psi(r, \theta, \varphi)=\sum_{n=0}^{n=\infty} h_{n}^{(1)}(k r) \sum_{m=0}^{m=n}\left(\alpha_{n m} \cos (m \varphi)+\beta_{n m} \sin (m \varphi)\right) P_{m}^{n}(\cos (\theta))
$$

où $h_{n}^{(1)}(z)=j_{n}(z)+i y_{n}(z)$ sont les fonctions de Hankel sphériques de première espèce et $P_{n}^{m}(z)$ est la fonction de Legendre de degré $n$ et d'ordre $m$. Pour déterminer les coefficients de la représentation de $\psi(M)$ en série d'harmoniques sphériques, la suite $\left(\alpha_{n m}, \beta_{n m}\right)$ est cherchée comme la solution du problème de minimisation suivant:

trouver $\alpha_{n m}$ et $\beta_{n m}\left(n=n_{1}, \ldots, n_{2} m=0, \ldots, n\right)$ tels que la forme quadratique :

$$
J=\sum_{i=1}^{i=N}\left|\psi\left(\alpha_{n m}, \beta_{n m} ; r_{i}, \theta_{i}, \varphi_{i}\right)-\tilde{\psi}\left(M_{i}\right)\right|^{2} \text { soit minimale. }
$$

Dans cette équation, $M_{i}(i=1,2, \ldots, N)$ est 1 'ensemble des points de mesure. répartis sur $\Sigma$. $\psi\left(\alpha_{n m}, \beta_{n m} ; r_{i}, \theta_{i}, \varphi_{i}\right)$ représente la série (1) réduite aux termes $n_{1}$ et $n_{2} \cdot\left(r_{i}, \theta_{i}, \varphi_{j}\right)$ sont les coordonnées des points $M_{j}$.

\subsection{Représentation de Green du champ acoustique rayonné par une plaque mince}

Soit une structure vibrante qui occupe un domaine $\Gamma$, de frontière $\partial \Gamma$. Cette source rayonne en espace indéfini $\Omega$ une pression acoustique $\psi(M)$, harmonique $\left(e^{-i \omega t}\right)$. Le fluide qui entoure la structure est supposé parfait. La pression acoustique est solution du système d'équations suivant :

$$
\begin{cases}\left(\Delta+k^{2}\right) \psi(M)=0 & \text { dans } \Omega \\ \partial_{\vec{n}} \psi\left(M^{\prime}\right)=\omega^{2} \rho_{f} u & \text { sur } \Gamma \\ \text { conservation de l'énergie } & \end{cases}
$$

où $\omega$ est la pulsation, $u$ le déplacement normal de la plaque et $\rho_{f}$ la densité du fluide. La condition de continuité des vitesses normales impose un gradient de la pression continu à la traversée de la plaque, le champ acoustique rayonné par la plaque $\psi(M)$ peut être alors représenté par un potentiel de double couche : 


$$
\psi(M)=\int_{\Gamma} \mu\left(M^{\prime}\right) \partial_{\vec{n}_{M}} G\left(M: M^{\prime}\right) d M^{\prime}
$$

où $\mu\left(M^{\prime}\right)$ est la densité de double couche. $G\left(M: M^{\prime}\right)$ est le noyau de Green de l'équation de Helmholtz de l'espace $\Omega$. Soit le point $M$ ' repéré dans le plan de la plaque par ses coordonnées polaires $M^{\prime}=\left(\rho^{\prime}, \theta^{\prime}\right)$. De part la géométrie de révolution du système physique. la densité de double couche $\mu\left(M^{\prime}\right)$ est cherchée sous forme d'une série de Fourier par rapport à la variable $\theta^{\prime}$. Le coefficient de chaque harmonique angulaire est cherché sous la forme d'un développement polynômial, on obtient :

$$
\mu\left(\rho^{\prime}, \theta^{\prime}\right)=\sum_{n=-\infty}^{n=+\infty} \sum_{m=0}^{m=+\infty} \alpha_{n m} P_{m}\left(\rho^{\prime}\right) e^{i n \theta^{\prime}} .
$$

La pression acoustique s'écrit alors, si $R$ est le rayon de la plaque:

$$
\psi(M)=\sum_{n=-\infty}^{n=+\infty} \sum_{m=0}^{m=+\infty} \alpha_{n m} A_{n m} \text {, où } A_{n m}=\int_{0}^{2 \pi} \int_{0}^{R} P_{m}\left(\rho^{\prime}\right) e^{i n \theta^{\prime}} \partial_{n_{M}}, G\left(M ; M^{\prime}\right) \rho^{\prime} d \rho^{\prime} d \theta^{\prime} \text {. }
$$

Le déplacement est $u\left(x^{\prime}, y^{\prime}\right)=u\left(\rho^{\prime}, \theta^{\prime}\right)=\omega^{2} \rho_{f} u=\partial_{z} \cdot \psi\left(x^{\prime}, y^{\prime}, z^{\prime}\right) z^{\prime}=0$. Il est clair qu'estimer le déplacement de la plaque revient a déterminer la série (4), donc les coefficients $\alpha_{n m}$. Ceux-ci sont obtenus en minimisant le carré de la norme $L^{2}$ de la différence entre la pression théorique et la pression mesurée. Sous forme discrète. cela revient à minimiser :

$$
F=\sum_{j=1}^{j=N m e s}\left|\sum_{n=-N}^{n=N} \sum_{m=0}^{m=M} \alpha_{n m} A_{n m}\left(M_{j}\right)-\tilde{\psi}\left(M_{j}\right)\right|^{2}
$$

expression dans laquelle $\tilde{\Psi}\left(M_{j}\right)$ représente la mesure de pression au capteur $j$, $\psi\left(\alpha_{n, m}, M_{j}\right)$ est la série (5), prise au point $M_{j}$ et Nmes est le nombre de mesures. I1 est évident que l'on n'a accès qu'a un nombre fini de mesures. Le nombre maximum de termes de Ta série (4) qu' il est possible déterminer est donné par Nmes $>(2 N+1)(M+1)$. La formule (4) est valable pour tout développement polynômial $P_{m}\left(\rho^{\prime}\right)$. Toutefois, non seulement, il est préférable de disposer d'une base orthonormée mais en outre, ii est indispensable que cette base soit adaptée au calcul numérique. Nous avons choisi donc de mettre en oeuvre la base des polynômes de Tchebycheff de première espèce

Le déplacement est obtenu simplement à partir de $\mu(M)$. La condition de continuité des vitesses normales donne:

$$
u(P)=\frac{1}{\omega^{2} \rho_{f}} \partial_{\vec{n}_{P}} \psi(P)=\frac{1}{\omega^{2} \rho_{f}} \operatorname{Pf} \int_{\Gamma} \mu\left(M^{\prime}\right) \partial_{n_{P}} \partial_{\vec{n}_{M}} G\left(P ; M^{\prime}\right) d M^{\prime},
$$

où Pf désigne la partie finie de l'intégrale. D'un point de vue numérique, il est nécessaire de procéder à une intégration analytique autour du point $P$. Pratiquement [2], on "isole" une petite surface autour de ce point. Une petite surface est telle que les produits de ses dimensions (de rapport voisin de 7 'unité) par le nombre d'onde soient petits. Soit $\sigma$ cette surface, on peut montrer que, si a est le rayon d'un disque de surface équivalente à la surface de $\sigma$, le déplacement est donné par :

$$
u(P)=\frac{1}{\omega^{2} \rho_{f}}\left\{\int_{\Gamma-\sigma} \mu\left(M^{\prime}\right) \partial_{n_{p}} \partial_{n_{M}} G\left(P ; M^{\prime}\right) d M^{\prime}+\frac{\mu(P)}{2}\left(\frac{e^{i k a}}{a}-i k\right)\right\} .
$$

\subsection{Solution exacte pour une plaque libre}

Dans le cas particulier d'une plaque circulaire à bords libres, une solution analytique [3] s'exprime comme une série de Fourier de fonction de Bessel. I] est clair que $n=0$ représente le mode de vibrations axisymétriques, le mode $n$ représente le mode de vibration à $n$ diamètres modaux. S représente le nombre de cercle modaux.

\section{RESULTATS ET COMMENTAIRES}

Il est très important de noter que si le nombre de mesures semble suffisant, du moins aux moyennes fréquences, pour une identification de sources multipolaires, if 
n'en est pas de même lorsque l'on s'interesse à la reconstitution du déplacenent. Pour chaque harmonique de la série de Fourier de la densité de double couche, on cherche à identifier un polynôme. Or, les micros sont répartis de telle manière que, sur un rayon, on dispose d'une ou de trois mesures. Pour une déformée radiale qui comporte deux minima (mode $s=2$ ), un polynôme de degré au moins égal à deux est nécessaire. Pour les modes supérieurs, 17 apparaît manisfestement un sous-échantillonage radial. Pour pal7ier cet inconvénient, nous avons reconstruit un champ de "mesures" à partir de la décomposition en série d'harmonique sphériques. Ce champ, qui comporte 114 mesures calculées par (1), présente un répartition radiale de 8 mesures. L'identification a été menée sur les deux champs de pression. Les résultats sont présentés figures 1 -a et 1-b pour un mode vibratoire $n=0, s=4$. Ce mode correspond à une fréquence de $1240 \mathrm{~Hz}$. L'identification a été menée avec un polynôme de Tchebycheff de degré 5 et un seut terme de la série de Fourier, figure 1-a, l'identification est menée pour le champ à 32 points et figure 1-b, elie est menée sur le champ à 114 points. Le trait plein représente la solution analytique, les triangles matérialisent les minima de déplacement mesurés. Il est tout à fait évident sur ces résultats que le l'identification sur le champ à 32 points échoue complètement alors que celle menée sur le champ interpolé donne des résultats tout à fait acceptables. Les figures 2 présentent la recontruction de la plaque lorsque celle-ci présente une discontinuité de masse (un accéléromètre). La figure 2-a donne une figure de Chladni recontruite à partir du champ à 114 points de mesure pour un mode vibratoire dont la fréquence correspond à $n=0, s=3$. Te déplacement est décrit par trois termes de la série de Fourier, chaque terme est décrit par un polynôme de Tchebycheff de degré 5. La figure 2-b est une vue de la figure de Chladni expérimentale. Là encore, on constate un excellent accord entre le modèle et 1 'expérience.

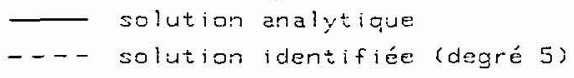

$\Delta$ Position cxpérimentale d'un minimum
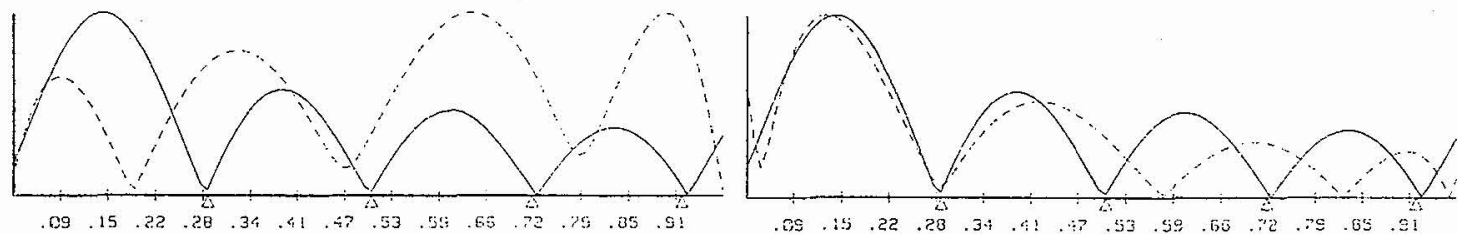

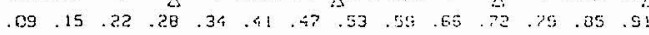

Figure 1-a

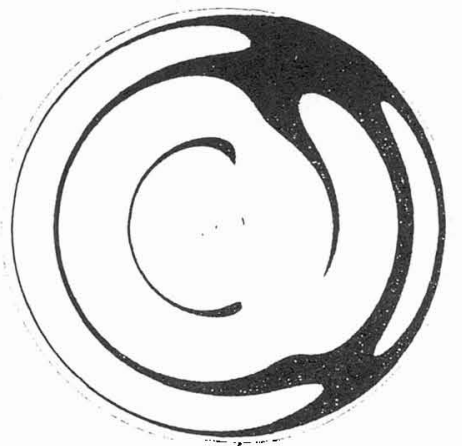

Figure 2-a
Figure 1-b

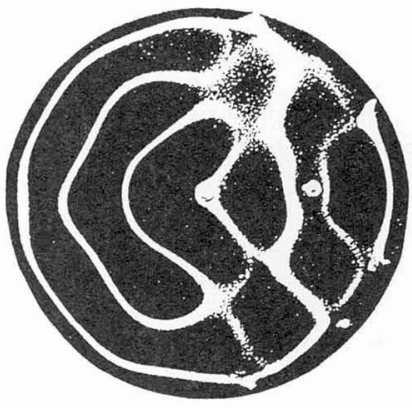

Figure 2-b

\section{REERENCES}

[1] P. Filippi, D. Habault and J. Piraux (1988). Journal of Sound and Vibration 124

(2), p. 285-296.

[2] F. Cassot (1971). Contribution à T'étude de la diffraction par un écran mince.

Thèse de spécialité en Acoustique. Marseille.

[3] A. W. Leissa (1969). Vibration of plates. NASA SP-160. 